\title{
Analysis of psychiatrists' prescription of opioid, benzodiazepine, and buprenorphine in Medicare Part $D$ in the United States
}

\author{
Kevin Pan, ${ }^{1}$ (D) Shawgi Silver, ${ }^{2}$ Charles Davis ${ }^{3}$
}

\begin{abstract}
Introduction: The opioid epidemic is a severe problem in the world, especially in the United States, where prescription opioid overdose accounts for a quarter of drug overdose deaths.

Objective: To describe psychiatrists' prescription of opioid, benzodiazepine, and buprenorphine in the United States.

Methods: We conducted a retrospective cross-sectional study of the 2016 Medicare Part D claims data and analyzed psychiatrists' prescriptions of: 1) opioids; 2) benzodiazepines, whose concurrent prescription with opioids can cause overdose death; 3 ) buprenorphine, a partial opioid agonist for treating opioid addiction; 4) and naltrexone microsphere, a once-monthly injectable opioid antagonist to prevent relapse to opioid dependence. Prescribers with 11 or more claims were included in the analysis.

Results: In Medicare Part $D$ in 2016, there were a total of $1,131,550$ prescribers accounting for $1,480,972,766$ total prescriptions and $78,145,305$ opioid prescriptions, including 25,528 psychiatrists ( $2.6 \%$ of all prescribers) accounting for $44,684,504$ total prescriptions ( $3.0 \%$ of all prescriptions) and 131,115 opioid prescriptions ( $0.2 \%$ of all opioid prescriptions). Psychiatrists accounted for $17.3 \%$ of benzodiazepine, $16.3 \%$ of buprenorphine, and $33.4 \%$ of naltrexone microsphere prescriptions. The opioid prescription rate of psychiatrists was much lower than that of all prescribers ( $0.3 \mathrm{vs} 5.3 \%)$. The buprenorphine prescription rate of psychiatrists was much higher than that of all prescribers (2.3 vs. $0.1 \%)$. There was a substantial geographical variation across the United States.

Conclusions: The results show that, proportionally, psychiatrists have lower rates of opioid prescription and higher rates of benzodiazepine and buprenorphine prescription.

Keywords: Psychiatry, addiction, geriatric psychiatry, opioid, addiction treatment.
\end{abstract}

\section{Introduction}

The opioid abuse crisis is a dangerous and growing problem in many parts of the world. ${ }^{1-3}$ For example, in Brazil, while the drug monitoring program has been successful at keeping opioid prescriptions $10{ }^{4}{ }^{4}$ the sales of prescription opioids still increased by more than $450 \%$ between 2009 and $2015 .^{5}$ The opioid epidemic is especially significant in the United States, where opioid overdose deaths account for more than $60 \%$ of all drug overdose deaths. ${ }^{6}$ In addition, $40 \%$ of the opioid overdose deaths are due to prescription opioids. ${ }^{7}$

As nations around the world search for ways to curb the opioid crisis, it would be interesting to examine the role of psychiatrists in this scenario. Psychiatrists, among all specialties, should have a minimal contribution

\footnotetext{
${ }^{1}$ Department of Economics, Finance, and Quantitative Analysis, Samford University, Birmingham, AL, USA. ${ }^{2}$ Department of Child and Adolescent Psychiatry, University of Washington, Seattle, WA, USA. ${ }^{3}$ Christ Health, Birmingham, AL, USA.

Submitted Mar 01 2019, accepted for publication Jun 172019.

Suggested citation: Pan K, Silver S, Davis C. Analysis of psychiatrists' prescription of opioid, benzodiazepine, and buprenorphine in Medicare Part D in the United States. Trends Psychiatry Psychother. 2020;42(1):48-54. http://dx.doi.org/10.1590/2237-6089-2019-0015
} 
to opioid prescription. However, it would be relevant to know if psychiatrists contribute to the opioid crisis indirectly. An important factor in opioid overdose death is the concurrent prescription of benzodiazepine, one of the most frequently prescribed medications for anxiey. ${ }^{8}$ Concurrent use of benzodiazepine and opioid can exacerbate overdose death, and the overdose mortality rate has increased drastically in the last two decades, with increasing concurrent prescriptions of opioid and benzodiazepine. ${ }^{9,10}$

In the study of opioid and benzodiazepine prescriptions, one particular population of interest is the elderly population, because of the increased prescriptions of benzodiazepines in this age group. ${ }^{8}$ Additionally, with the elderly patients susceptible to suicide, ${ }^{11}$ there is a dangerous association between opioid abuse and suicidal attempts in the geriatric patient population: geriatric patients with suicidal ideation can consume more opioid and benzodiazepine, which could result in overdose death. ${ }^{12-14}$

In the United States, many of the elderly patients are covered by Medicare, an insurance plan for Americans who are 65 years or older and patients with certain disabilities. A particular part of Medicare is Medicare Part $D$, which is an optional coverage plan for prescription drugs. When a beneficiary becomes eligible, he or she can choose to enroll in the Medicare Part D plan. ${ }^{15}$ Once a beneficiary is enrolled, qualified prescription medications can be covered by the plan. Even though it is optional, many patients choose to enroll in it: more than 43 million beneficiaries are enrolled in the optional Medicare Part D today, accounting for more than 1 billion prescriptions and USD 80 billion in claims each year. ${ }^{16}$ Of the total prescriptions written for Medicare Part D patients, more than 78 million were written for opioids in 2016. ${ }^{17}$ Furthermore, more than 6 of every 1,000 Medicare beneficiaries have opioid use disorder, which is among the highest rates considering all patient groups. ${ }^{18}$

Meanwhile, psychiatrists could play an important role in the treatment of opioid addiction. One approach is to treat opioid addiction using buprenorphine, a partial opioid receptor agonist that has been shown to be an effective maintenance treatment for opioid dependence. ${ }^{19}$ To be able to prescribe buprenorphine in the United States, a physician has to obtain a waiver from the Drug Enforcement Administration (DEA); among all physicians that have obtained a waiver to prescribe buprenorphine, $41.6 \%$ were psychiatrists. ${ }^{20}$ Previously, the relative ratio of opioid and opioid agonist treatment has been examined for each specialty, ${ }^{21}$ but the overall contribution of psychiatrists to all buprenorphine prescriptions was not reported.
In addition to buprenorphine, another approach to combat opioid addiction is extended-release naltrexone, formulated as naltrexone microspheres, a monthly injectable opioid antagonist. ${ }^{22}$ Previous data showed that buprenorphine and extended-release naltrexone had similar safety and effectiveness, once initiated. ${ }^{23}$

In this paper, we seek to describe psychiatrists' prescriptions of opioid, benzodiazepine, buprenorphine and naltrexone microspheres using the Medicare Part D Provider Utilization and Payment Data for year 2016. We examine the percentage of prescriptions coming from psychiatrists for each of these medications, and also analyze the geographical distribution of the percentages.

\section{Methods}

Following Chen et al., ${ }^{16}$ we used the Medicare Provider Utilization and Payment Data: Part D for our analysis. ${ }^{24}$ The study was considered by the university's institutional review board to be exempt from approval, as it only used existing publicly available data. This study was a retrospective, cross-sectional study: we were interested in having a cross-sectional view of opioid, benzodiazepine, and buprenorphine prescriptions in the United States. For the cross-sectional analysis, we used the most recent year then available, which was year 2016.

For our analysis, we adopted the same list of opioids defined by the Medicare Provider Utilization and Payment Data, which contains 93 opioids, including fentanyl, hydrocodone, hydromorphone, methadone, morphine, oxycodone, oxymorphone, and tramadol. ${ }^{24}$ The complete list of opioids is available at the Medicare Provider Utilization and Payment Data website. ${ }^{24}$ Benzodiazepines include clonazepam, lorazepam, and alprazolam.

We followed the same inclusion and exclusion criteria defined by the Medicare Provider Utilization and Payment Data, i.e., prescribers with fewer than 11 claims were excluded from analysis; only prescribers with 11 or more claims were included. ${ }^{24}$ Therefore, in order to be considered an opioid prescriber, at least 11 opioid claims had to be found on the system for that presciber. These inclusion and exclusion criteria helped to protect identifiable patient information.

We calculated ratios representing total drug claims per prescriber and opioid claims per prescriber, as follows:

$$
\text { All Claims Per Prescriber }=\frac{\sum \text { Total Claim Count }}{\text { Number of Prescribers }}
$$




$$
\text { Opioid Claims Per Prescriber }=\frac{\sum \text { Opioid Claim Count }}{\text { Number of Prescribers }}
$$

For each prescriber specialty, the opioid prescription was calculated as follows 24 :

$$
\text { Opioid Prescription Rate }=\frac{\text { Opioid Claim Count }}{\text { Total Claim Count }}
$$

All the analyses in this study refer to population statistics, as we examined the complete set of Medicare Part D data, which included all providers in Medicare Part D. As a result, all numbers reported are population statistics, not sample statistics. Therefore, sample statistics measures such as $p$-values are not applicable in this study.

Analyses were performed using Microsoft Excel (Microsoft Inc.) and Tableau Desktop software (Tableau Inc.). Geographical distribution was analyzed using the location information available in Medicare Part D data, namely, prescriber state and city. This allowed us to find the number of claims among all prescribers in each state. Likewise, we were able to calculate the percentages of each state. Computationally, geographical analysis and charting were performed using Tableau Desktop software.

\section{Results}

\section{Opioid prescription analysis}

Table 1 shows the number and percentage of prescribers who were psychiatrists. There were a total of $1,131,550$ prescribers of all specialties in Medicare Part D, 2016. Among them, 25,528 were psychiatrists, accounting for $2.3 \%$ of all prescribers. In terms of opioid prescription claims, there were a total of $78,145,305$ claims among all prescribers. Psychiatrists accounted for only 131,115 opioid claims, i.e., $0.2 \%$ of all opioid claims. Table 1 also shows the opioid prescription rates of psychiatrists in comparison with all prescribers. Among all prescribers, $44.2 \%$ prescribed opioids, and the overall opioid prescription rate was 5.4\%. Among psychiatrists, $8.9 \%$ prescribed opioids, and the overall opioid prescription rate was $0.3 \%$.

Figure 1 shows the geographical distribution of the percentages of opioid claims accounted for by psychiatrists in the 48 states (Alaska and Hawaii are not shown in the figure but were included in the analysis) in the United States. The states with the highest percentages were West Virginia, Rhode Island, and Kansas. In these three states, psychiatrists accounted for $0.81 \%$ of all opioid prescriptions. In all other states, psychiatrists accounted for less than $0.33 \%$ of all opioid prescriptions.

\section{Benzodiazepine prescription analysis}

Table 1 also shows the number and percentage of benzodiazepines prescribed by psychiatrists among all prescribers in Medicare Part D in 2016. There were $33,640,456$ benzodiazepine claims, of which 5,820,447 were prescribed by psychiatrists. In other words, psychiatrists accounted for $17.3 \%$ of benzodiazepine claims. Table 1 also shows the benzodiazepine prescription rates of psychiatrists in comparison with all prescribers. Among all prescribers, 26.3\% prescribed benzodiazepines, and the overall benzodiazepine prescription rate was $2.6 \%$. Among psychiatrists, $82.2 \%$ prescribed benzodiazepines, and the overall benzodiazepine prescription rate was $14.0 \%$.

Figure 2 shows the geographical distribution of the percentages of benzodiazepine claims accounted for by psychiatrists in the 48 states (Alaska and Hawaii are not shown in the figure but were included in the analysis) in the United States. The states with the highest percentages were New York, Minnesota, Massachusetts,

Table 1 - Percentage of opioid and benzodiazepine prescriptions accounted for by psychiatrists, Medicare Provider Utilization and Payment Data: Part D, 2016

\begin{tabular}{lcc}
\hline & All specialties & $\begin{array}{c}\text { Psychiatrists (percentage of all } \\
\text { specialties) }\end{array}$ \\
\hline Total number of prescribers & $1,131,550$ & $25,528(2.3 \%)$ \\
Total number of all prescriptions & $1,480,972,766$ & $44,684,504(3.0 \%)$ \\
Total number of opioid claims & $78,145,305$ & $131,115(0.2 \%)$ \\
Percentage of prescribers who prescribe opioids & $44.2 \%$ & $8.9 \%$ \\
Opioid prescription rate & $5.3 \%$ & $0.3 \%$ \\
Total number of benzodiazepine claims & $33,640,456$ & $5,820,447(17.3 \%)$ \\
Percentage of prescribers who prescribe benzodiazepines & $26.3 \%$ & $82.2 \%$ \\
Benzodiazepine prescription rate & $2.6 \%$ & $14.0 \%$ \\
\hline
\end{tabular}

50 - Trends Psychiatry Psychother. 2020;42(1) 
Maryland, Wisconsin, and Rhode Island. Psychiatrists accounted for nearly $25 \%$ of all benzodiazepine prescriptions in these states.

\section{Buprenorphine prescription analysis}

Table 2 shows the number and percentage of buprenorphine prescriptions by psychiatrists among all prescribers in Medicare Part D in 2016. There were $1,007,115$ buprenorphine claims, of which 164,147 were prescribed by psychiatrists. In other words, psychiatrists accounted for $16.3 \%$ of buprenorphine claims. Table 2 also shows the buprenorphine prescription rates of psychiatrists in comparison with all prescribers. Among all prescribers, $1.4 \%$ prescribed buprenorphine, and the overall buprenorphine prescription rate was $0.1 \%$. Among psychiatrists, $7.8 \%$ prescribed buprenorphine, and the overall buprenorphine prescription rate was $2.3 \%$.

Figure 3 shows the geographical distribution of the percentages of buprenorphine claims accounted for by



Percentage

$0.02 \% \quad 0.81 \%$

Figure 1 - Percentage of opioids prescribed by psychiatrists among all prescribers for the states in the United States (Alaska and Hawaii not shown but accounted for), Medicare Provider Utilization and Payment Data: Part D, 2016.



\section{Percentage}

$5.47 \% \quad 25.03 \%$

Figure 2 - Percentage of benzodiazepines prescribed by psychiatrists among all prescribers for the states in the United States (Alaska and Hawaii not shown but accounted for), Medicare Provider Utilization and Payment Data: Part D, 2016. 
psychiatrists in the 48 states (Alaska and Hawaii are not shown in the figure but were included in the analysis) in the United States. The states with the highest percentages were Hawaii, Connecticut, Wyoming, and West Virginia, where psychiatrists accounted for more than $30 \%$ of all buprenorphine prescriptions.

\section{Naltrexone microsphere prescription analysis}

Table 2 also shows the number and percentage of naltrexone microsphere prescriptions by psychiatrists among all prescribers in Medicare Part D in 2016. There were 4,357 naltrexone microsphere claims, and 1,455 of them were prescribed by psychiatrists. In other words, psychiatrists accounted for $33.4 \%$ of naltrexone microsphere claims. Due to the small size of data, geographical analysis of naltrexone microsphere prescriptions was not performed.

\section{Discussion}

As countries around the world strategize to combat the opioid crisis, psychiatrists can play a pivotal role in this battle. First, psychiatrists contribute directly, even if minimally, to opioid prescriptions. As shown in the present study, psychiatrists account for $2.3 \%$ of all prescribers, and yet only $0.2 \%$ of all opioid claims. This is not surprising, as psychiatrists do not practice pain management as often as physicians from other specialties.

While psychiatrists have a minimal direct contribution to opioid prescriptions, they could help reduce the number of opioid overdose deaths by monitoring benzodiazepine prescriptions. In other words, while psychiatrists do not prescribe opioids to patients directly, the same patients could be receiving opioids from other physicians.

Table 2 - Percentage of buprenorphine and naltrexone microsphere prescriptions accounted for by psychiatrists, Medicare Provider Utilization and Payment Data: Part D, 2016

\begin{tabular}{|c|c|c|}
\hline & All specialties & $\begin{array}{c}\text { Psychiatrists (percentage of all } \\
\text { specialties) }\end{array}$ \\
\hline Total number of prescribers & $1,131,550$ & $25,528(2.3 \%)$ \\
\hline Total number of all prescriptions & $1,480,972,766$ & $44,684,504(3.0 \%)$ \\
\hline Total number buprenorphine claims & $1,007,115$ & $164,147(16.3 \%)$ \\
\hline Percentage of prescribers who prescribe buprenorphine & $1.4 \%$ & $7.8 \%$ \\
\hline Buprenorphine prescription rate & $0.1 \%$ & $2.3 \%$ \\
\hline Total number of naltrexone microsphere claims & 4,357 & $1,455(33.4 \%)$ \\
\hline Percentage of prescribers who prescribe naltrexone microsphere & $0.022 \%$ & $0.33 \%$ \\
\hline Naltrexone microsphere prescription rate & $0.0003 \%$ & $0.0033 \%$ \\
\hline
\end{tabular}

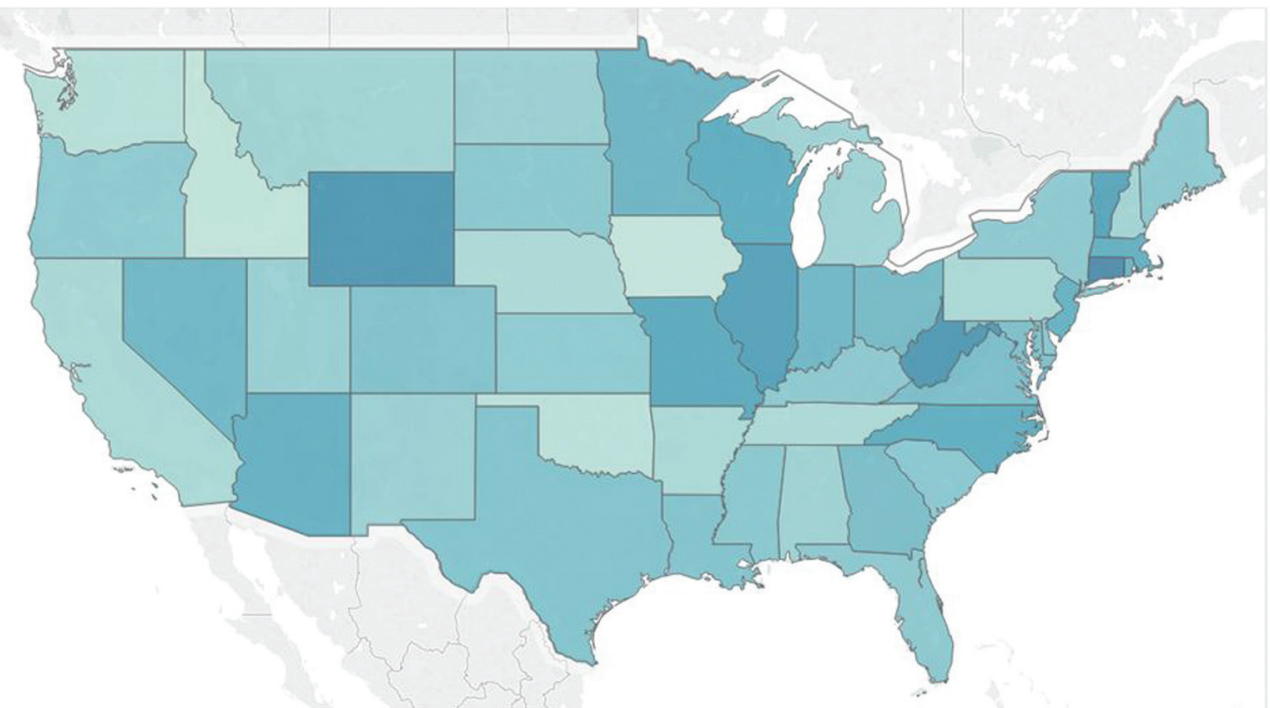

Percentage

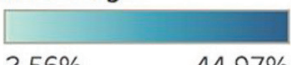

$2.56 \%$

$44.97 \%$

Figure 3 - Percentage of buprenorphine prescriptions by psychiatrists among all prescribers for the states in the United States (Alaska and Hawaii not shown but accounted for), Medicare Provider Utilization and Payment Data: Part D, 2016. 
Therefore, when psychiatrists prescribe benzodiazepine, it would be beneficial to ask their patients if they are receiving opioids from other physicians, concurrently with the benzodiazepine prescription. In this paper, we did not analyze whether a patient received opioids and benzodiazepines simultaneously because patientlevel data were not available (only prescriber-level data were). To protect patient confidentiality, no patient-level data or prescription-level data were available. Future studies should investigate concurrent prescription of opioids and benzodiazepines.

Likewise, we were also not able to study patient demographics or clinical indications associated with the prescriptions, since such data were not available. Similarly, in the Medicare Part D data, there were no cases of duplicate claims at prescriber level, but there could be duplicate prescription claims at patient level, i.e., the same patient receiving multiple prescriptions from different prescribers. Future research could use smaller-scale studies with private data to study these factors.

Further, psychiatrists can help in the fight against the opioid crisis by prescribing opioid addiction treatments, including buprenorphine and naltrexone microspheres. While psychiatrists already contribute significantly to the prescription of buprenorphine, it seems that they could contribute even more, because only about $16 \%$ of psychiatrists have a waiver from the DEA to prescribe buprenorphine, ${ }^{20}$ and only $7.8 \%$ of psychiatrists prescribe buprenorphine (Table 2). Also, it seems that naltrexone microspheres account for a small amount of prescriptions in Medicare Part D, yet this is a valid treatment option for psychiatrists and their patients to consider to help prevent relapse. A next step could be to encourage more psychiatrists to receive provider education on buprenorphine and naltrexone microspheres, and to investigate why some of those who have received the DEA waiver are not actively prescribing buprenorphine.

Previous literature has suggested that psychiatrists are more concentrated in urban areas, and therefore do not contribute as much to buprenorphine prescriptions in rural areas. ${ }^{20}$ However, our results showed that this is not necessarily the case: in more rural states, such as West Virginia and Wyoming, psychiatrists contributed to more than $30 \%$ of all buprenorphine prescriptions. In rural areas, primary care physicians could prescribe opioids more frequently than their peers in urban areas. ${ }^{25}$ To combat the opioid crisis in the rural areas, psychiatrists can likely play a key role in opioid addiction treatment.

Our results suggest that there is substantial geographical variation for the prescription of both benzodiazepine (Figure 2) and buprenorphine (Figure 3) by psychiatrists. A recent paper suggested that the opioid epidemic is not limited to rural areas or the Midwestern states, as previously thought, but has migrated toward urban and eastern states. ${ }^{26}$ Our analysis of the geographical distribution suggested that there are states, such as New York, where psychiatrists could monitor benzodiazepine prescriptions, as they account for almost $25 \%$ of all benzodiazepine prescriptions, and they could also study the possibility of increasing prescription of opioid addiction treatments such as buprenorphine, as the percentages in these states are lower than in other states.

This study was a retrospective, cross-sectional study. For future research, a longitudinal analysis could be performed to characterize the trend of opioid, benzodiazepine, and buprenorphine prescriptions over time. For instance, it would be of interest to know if buprenorphine prescriptions become more prevalent. Also, a future prospective study could assess the efficacy of opioid treatment and addiction prevention by psychiatrists.

By combining opioid treatment medications with other modalities of addiction treatment, psychiatrists can play an important role in reducing the opioid epidemic. It was previously reported that patients with cocaine dependence can have depression and anxiety. ${ }^{27}$ It is possible that patients with opioid dependence can similarly have depression and anxiety, and therefore psychiatric treatments should be an important part of addiction treatment. For elderly patients, a holistic approach, including other activities such as physical exercise, can help patients cope with anxiety. ${ }^{28}$ Similarly, to treat anxiety, holistic quality of life assessment should be considered. ${ }^{29}$

Currently, whenapsychiatristprescribesbuprenorphine or naltrexone microspheres, there is no clear financial benefit to the prescriber, as a psychiatrist likely gets paid similar amounts either prescribing buprenorphine or providing typical psychiatric services. However, for a psychiatrist to be able to prescribe buprenorphine, they have to afford additional expenses, such as obtaining the waiver from the DEA, ${ }^{20}$ training, record keeping, and overhead costs. Therefore, psychiatrists may not be incentivized to offer buprenorphine. Meanwhile, there are grants for community health centers in the United States to help support medication-assisted treatment such as with buprenorphine. ${ }^{30}$ One possible future policy change could be to offer grants for psychiatrists to provide medication-assisted treatment for opioid abuse. ${ }^{31}$ Future studies could investigate the cost-effectiveness of awarding such grants to psychiatrists to help combat the opioid crisis. 


\section{Acknowledgements}

This study received financial support from the Hull Fund Christian Scholarship Grant at Samford University, Birmingham, AL, USA.

\section{Disclosure}

No conflicts of interest declared concerning the publication of this article.

\section{References}

1. Scherrer JF, David Schneider F, Lustman PJ. Opioid analgesics and chronic non-cancer pain: a prescription for research in primary care. Fam Pract. 2016;33:569-71.

2. Dyer O. Canada's prescription opioid epidemic grows despite tamperproof pills. BMJ. 2015;351:h4725.

3. Mordecai L, Reynolds C, Donaldson LK, Williams AC. Patterns of regional variation of opioid prescribing in primary care in England: a retrospective observational study. $\mathrm{Br}$ J Gen Pract. 2018;68:e225-33.

4. Pacurucu-Castillo SF, Ordóñez-Mancheno JM, Hernández-Cruz A, Alarcón RD. World opioid and substance use epidemic: a Latin American perspective. Psychiatr Res Clin Pract. 2019;1:32-8.

5. Krawczyk N, Greene MC, Zorzanelli R, Bastos FI. Rising trends of prescription opioid sales in contemporary Brazil, 2009-2015. Am J Public Health. 2018;108:666-8.

6. Rudd RA, Seth P, David F, Scholl L. Increases in drug and opioidinvolved overdose deaths - United States, 2010-2015. MMWR Morb Mortal Wkly Rep. 2016;65:1445-52.

7. Seth $P$, Scholl L, Rudd RA, Bacon S. Overdose deaths involving opioids, cocaine, and psychostimulants - United States, 20152016. MMWR Morb Mortal Wkly Rep. 2018;67:349-58.

8. Schallemberger JB, Colet Cde F. Assessment of dependence and anxiety among benzodiazepine users in a provincial municipality in Rio Grande do Sul, Brazil. Trends Psychiatry Psychother. 2016;38:63-70.

9. Bachhuber MA, Hennessy S, Cunningham CO, Starrels JL. Increasing benzodiazepine prescriptions and overdose mortality in the United States, 1996-2013. Am ] Public Health, 2016:106:686-8.

10. Sun EC, Dixit A, Humphreys K, Darnall BD, Baker LC, Mackey $\mathrm{S}$. Association between concurrent use of prescription opioids and benzodiazepines and overdose: retrospective analysis. BMJ. 2017;356:j760.

11. Santos ADD, Guimarães LML, Carvalho YF, Viana LDC, Alves GL, Lima ACR, et al. Spatial analysis and temporal trends of suicide mortality in Sergipe, Brazil, 2000-2015. Trends Psychiatry Psychother. 2018;40:269-76.

12. West NA, Severtson SG, Green JL, Dart RC. Trends in abuse and misuse of prescription opioids among older adults. Drug Alcohol Depend. 2015;149:117-21.

13. Maree RD, Marcum ZA, Saghafi E, Weiner DK, Karp JF. A systematic review of opioid and benzodiazepine misuse in older adults. Am J Geriatr Psychiatry. 2016;24:949-63.

14. Schepis TS, Simoni-Wastila L, McCabe SE. Prescription opioid and benzodiazepine misuse is associated with suicidal ideation in older adults. Int J Geriatr Psychiatry. 2019;34:122-9.

15. U.S. Department of Health \& Human Services. Drug coverage (Part D). [cited 2018 Dec 15]. https://www.medicare.gov/drugcoverage-part- $d$
16. Chen JH, Humphreys $\mathrm{K}$, Shah $\mathrm{NH}$, Lembke A. Distribution of opioids by different types of Medicare prescribers. JAMA Intern Med. 2016:176:259-61.

17. U.S. Department of Health \& Human Services, Office of Inspector General. Opioids in Medicare Part D: concerns about extreme use and questionable prescribing. HHS OIG Data Brief. 2017;1-16.

18. Dufour R, Joshi AV, Pasquale MK, Schaaf D, Mardekian J, Andrews GA, et al. The prevalence of diagnosed opioid abuse in commercial and Medicare managed care populations. Pain Pract. 2014;14:E106-15.

19. Mattick RP, Kimber J, Breen C, Davoli M. Buprenorphine maintenance versus placebo or methadone maintenance for opioid dependence. Cochrane Database Syst Rev. 2004:CD002207.

20. Rosenblatt RA, Andrilla CHA, Catlin M, Larson EH. Geographic and specialty distribution of US physicians trained to treat opioid use disorder. Ann Fam Med. 2015;13:23-6.

21. Lembke $A$, Chen JH. Use of opioid agonist therapy for Medicare patients in 2013. JAMA Psychiatry. 2016;73:990-2.

22. Lee JD, Friedmann PD, Kinlock TW, Nunes EV, Boney TY, Hoskinson RA Jr, et al. Extended-release naltrexone to prevent opioid relapse in criminal justice offenders. N Engl J Med. 2016;374:1232-42.

23. Lee JD, Nunes EV, Novo P, Bachrach K, Bailey GL, Bhatt S, et al. Comparative effectiveness of extended-release naltrexone versus buprenorphine-naloxone for opioid relapse prevention (X:BOT): A multicentre, open-label, randomized controlled trial. Lancet. 2018;391:309-18.

24. Centers for Medicare and Medicaid Services. Medicare provider utilization and payment data: Part D prescriber. [cited 2018 Dec 15]. https://www.cms.gov/Research-Statistics-Data-andSystems/Statistics-Trends-and-Reports/Medicare-ProviderCharge-Data/Part-D-Prescriber.html

25. Pan K, Blankley AI, Hughes PJ. An examination of opioid prescription for Medicare Part $D$ patients among family practice prescribers. Fam Pract. 2019;36:467-72.

26. Kiang MV, Basu S, Chen J, Alexander MJ. Assessment of changes in the geographical distribution of opioid-related mortality across the United States by opioid type, 1999-2016. JAMA Netw Open. 2019;2:e190040.

27. de Oliveira LDSSCB, Souza EC, Rodrigues RAS, Fett CA, Piva AB. The effects of physical activity on anxiety, depression, and quality of life in elderly people living in the community. Trends Psychiatry Psychother. 2019;41:36-42.

28. Schallemberger JB, Colet Cde F. Assessment of dependence and anxiety among benzodiazepine users in a provincial municipality in Rio Grande do Sul, Brazil. Trends Psychiatry Psychother. 2016;38:63-70.

29. Schwab B, Daniel HS, Lutkemeyer C, Neves JA, Zilli LN, Guarnier $\mathrm{R}$, et al. Variables associated with health-related quality of life in a Brazilian sample of patients from a tertiary outpatient clinic for depression and anxiety disorders. Trends Psychiatry Psychother. 2015;37:202-8.

30. The United States Department of Health and Human Services. HHS awards over $\$ 1$ billion to combat the opioid crisis. [Cited 2019 Jun 3]. https://www.hhs.gov/about/news/2018/09/19/hhsawards-over-1-billion-combat-opioid-crisis.html

31. Joshi P, Shah NK, Kirane HD. Medication-assisted treatment for opioid use disorder in older adults: an emerging role for the geriatric psychiatrist. Am J Geriatr Psychiatry. 2019;27:455-7.

\section{Correspondence:}

Kevin Pan

Department of Economics, Finance, and Quantitative Analysis Brock School of Business, Samford University

800 Lakeshore Dr.

35229 - Birmingham, AL - USA

Tel. : +1 (205) 7264640

E-mail:kpan@samford.edu 